\title{
Confined Electroconvective Vortices at Structured Ion Exchange Membranes
}

\author{
Joeri de Valença, ${ }^{\dagger \dagger}$ Morten Jõgi, ${ }^{\ddagger}$ R. Martijn Wagterveld, ${ }^{\ddagger}$ Elif Karatay, ${ }^{\S}$ Jeffery A. Wood, ${ }^{\dagger}$ \\ and Rob G. H. Lammertink*,† \\ ${ }^{\dagger}$ Soft Matter, Fluidics and Interfaces Group, MESA ${ }^{+}$Institute of Nanotechnology, University of Twente, 7500AE Enschede, The \\ Netherlands \\ ${ }^{*}$ Wetsus, European Centre of Excellence for Sustainable Water Technology, Oostergoweg 9, 8911MA Leeuwarden, The Netherlands \\ ${ }^{\S}$ Department of Mechanical Engineering, Stanford University, Stanford, California 94305, United States
}

\section{Supporting Information}

ABSTRACT: In this paper, we investigate electroconvective ion transport at cation exchange membranes with different geometry square-wave structures (line undulations) experimentally and numerically. Electroconvective microvortices are induced by strong concentration polarization once a threshold potential difference is applied. The applied potential required to start and sustain electroconvection is strongly affected by the geometry of the membrane. A reduction in the resistance of approximately $50 \%$ can be obtained when the structure size is similar to the mixing layer (ML) thickness, resulting in confined vortices with less lateral motion compared to the case of flat membranes. From electrical, flow, and concentration measurements, ion migration, advection, and diffusion are quantified, respectively. Advection and migration are dominant in the vortex ML, whereas diffusion and migration are dominant in the stagnant diffusion layer. Numerical simulations, based on Poisson-Nernst-Planck and Navier-Stokes equations, show similar ion transport and flow characteristics, highlighting the importance of membrane topology on the resulting electrokinetic and electrohydrodynamic behavior.

\section{INTRODUCTION}

Electroconvection (EC) in electrolyte solutions can occur when a sufficiently strong electric field oriented perpendicular to a charge-selective interface (ion exchange membrane, ${ }^{1}$ electrode, ${ }^{2}$ microchannel, nanochannel, ${ }^{3}$ etc.) is applied. EC is caused by an electro-kinetic instability (EKI) of the fluid near the ionselective surface. On one hand, this allows faster ion transport than diffusion alone near the interface, thereby possibly enhancing the desalination rate in electrodialysis ${ }^{4}$ or the electrodeposition speed. ${ }^{5}$ On the other hand, to achieve this, mixing the current efficiency drops versus operating in the Ohmic regime and unwanted processes such as water-splitting ${ }^{4}$ or complex dendrite formation may occur. ${ }^{5}$

A cation exchange membrane (CEM) allows cations to migrate through while rejecting the anions that migrate in the opposite direction. The ion-flux imbalance at the interfaces results in concentration change: at one side of the membrane, the concentration enriches, and at the other side, it depletes. The development of concentration gradients in the aqueous solution is called ion concentration polarization (ICP). When the interface concentration at the depleted side approaches zero, the high electrical resistance of this depletion layer dominates the overall resistance. The corresponding limiting current $\left(i_{\text {lim }}\right)$ is seen as the upper limit of practical application in electrodialysis. ${ }^{6}$ If the voltage is increased further, the high resistance fluid layer eventually becomes unstable and additional current is observed, which is referred to as overlimiting current (OLC). The occurrence of OLC has been known for more than 50 years. ${ }^{7,8}$ Several physical and chemical mechanisms have been proposed to describe OLC, such as water-splitting or under electrodiffusion conditions in ac fields; however, interfacial mixing of the depleted boundary layer due to EC (electo-osmosis) is considered as a typical mechanism. ${ }^{1,4,8-10}$

For EC, there are many different potential mechanisms, which can arise depending on the system characteristics. ${ }^{11}$ Dukhin explored the coupling of an electric field along a curved surface in an electrolyte and described the formation of an extended space charge layer (ESCL). ${ }^{12,13}$ This layer forms when the concentration is depleted out of equilibrium by an external electric field. Rubinstein and Zaltzman worked out a theory on how the ESCL at a flat membrane could become unstable such that a small perturbation starts the electroosmosis parallel to the surface-forming vortices that enhance

Received: December 4, 2017

Revised: January 17, 2018

Published: January 18, 2018 
the initial inhomogeneity. ${ }^{10,14}$ This instability mechanism is referred to as the EKI. ${ }^{15,16}$ At an undulated surface, both the electroconvective mechanisms can occur. Rubinstein predicted that an undulated surface decreases the energy needed to sustain the vortices if the mixing layer $(M L)$ is of a dimension comparable to that of the undulations. ${ }^{10,17}$ Recent direct numerical simulations by Davidson et al. ${ }^{18}$ investigated a patterned surface consisting of patches of conducting and nonconducting surfaces. They reported a theoretical increase in ion transport of $80 \%$ for pattern sizes similar to the $\mathrm{ML}$ thickness.

Physical experiments with geometrically structured membranes have shown that a $60 \%$ decrease in voltage required for the onset of OLC can be obtained, determined solely using electrical characterization. ${ }^{19}$ Follow-up studies with chemically patterned membranes were found to also cause a reduction in the onset voltage. ${ }^{20}$ Chemical degradation of membranes was found to induce inhomogeneities at the surface that enhance the onset of EC. ${ }^{21}$ Additionally, hydrophobicity of the surface can influence the efficiency of EC. ${ }^{22}$ All previous experimental work regarding this phenomenon to date have relied on a purely electrical characterization of these systems. This paper extends the knowledge by combining the experimental observations of the electrical behavior with the simultaneous determination of the flow and concentration fields next to a membrane with periodic line structures. We show that the current efficiency of electroconvective mixing at a CEM increases when the size of the membrane undulations is of the same order as the ML thickness, stabilizing the position of the electroconvective vortices at the membrane during operation in the OLC regime.

\section{MATERIALS \& METHODS}

Membrane Preparation and Pretreatment. The CEMs are composed of 83 wt \% sulfonated poly ether ether ketone (SPEEK) mixed with 17 wt \% glycerol. To fabricate the membranes, SPEEK (67\% sulfonation degree), glycerol, ethanol, and ultrapure water (Milli-Q) were used. A mixture of $40 \mathrm{~g}$ of SPEEK and $8 \mathrm{~g}$ of glycerol is dissolved in $160 \mathrm{~g}$ water/ethanol (1:1). The solution is poured on a pattern-etched mold and sonicated in an ultrasound bath for $15 \mathrm{~min}$ to release trapped air bubbles. After the ultrasonic bath, it is placed under an $\mathrm{N}_{2}$ atmosphere for $24 \mathrm{~h}$, after which it can be peeled off the mold. The mold was made using a photolithography and reactive ion-etching process in the cleanroom. The process is similar to what was described previously by Balster et al. ${ }^{19}$ The structures were designed to have rectangular ridges with an extrusion height $(H)$ of $40 \mu \mathrm{m}$ and varying widths $(L)$ of $50,100,200,400$, and $800 \mu \mathrm{m}$; see Figure 1 . For all patterns, the gap has the same width as the extrusion ( $50 \%$ periodicity in the undulation).

To ensure complete ion exchange, the membranes are immersed in a $100 \mathrm{mM} \mathrm{CuSO}{ }_{4}$ solution for $24 \mathrm{~h}$. Next, the membranes are equilibrated with the electrolyte solution used for the measurements, $10 \mathrm{mM} \mathrm{CuSO}{ }_{4} \cdot 5 \mathrm{H}_{2} \mathrm{O}$ (VWR Chemicals) in ultrapure water (Milli-Q). Then, the membrane is rinsed with ultrapure water and the excess water is wiped off. Afterward the membrane is placed in the measurement solution for $24 \mathrm{~h}$. The solution is changed three times to ensure the membrane is in equilibrium with the measurement solution.

Experimental Setup. Figure 2 shows a schematic of the experimental setup with a structured membrane, with an area of $A_{\text {mem }}=3 \times 4.5 \mathrm{~mm}^{2}$, placed between two reservoirs filled with a 10 $\mathrm{mM} \mathrm{CuSO}$ electrolyte solution, enclosed by copper cathode and anode. The anode compartment has a thickness of $L_{\text {com }}=2 \mathrm{~mm}$. For extended details on the mounting of the cell, as well as for camera positions and other details, see Chapter 2 of de Valença. ${ }^{23}$ A voltage difference is applied between these two electrodes by a potentiostat

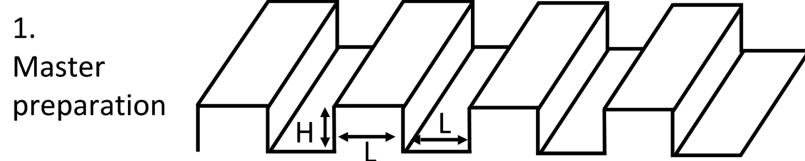

2.

Casting
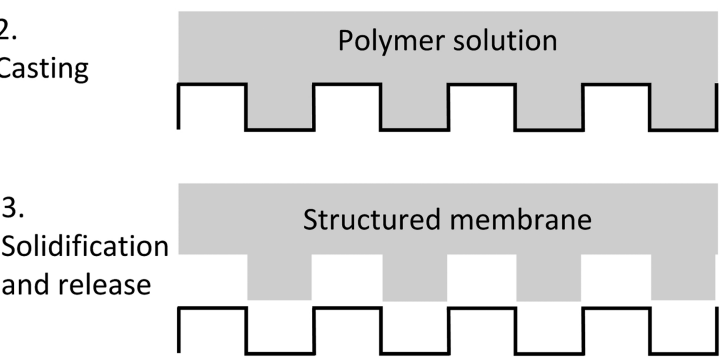

4.

SEM image

membrane

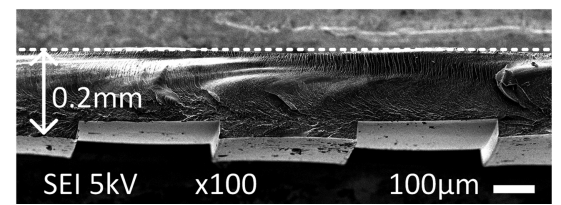

Figure 1. Fabrication process for structured membranes. A SPEEK solution is cast on a microstructured mold. After solvent evaporation, the membrane is solidified and released. Membranes with $L=50,100$, 200, 400, and $800 \mu \mathrm{m}$ and $H=50 \mu \mathrm{m}$ and a flat membrane were made. SEM image of a structured membrane with $L=400 \mu \mathrm{m}$. The dotted line represents the back side of the membrane.

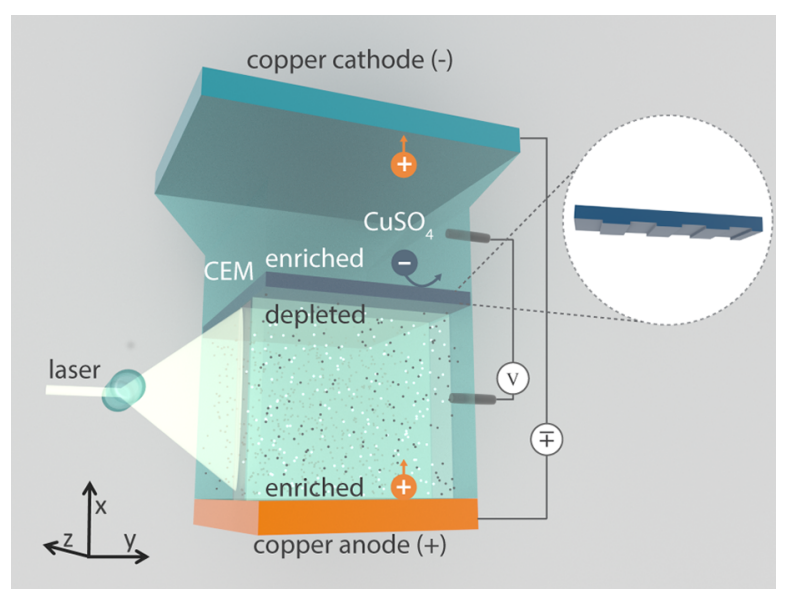

Figure 2. Structured membrane between the anode and cathode compartment filled with $\mathrm{CuSO}_{4}$ solution. The flow dynamics are captured by imaging the displacement of the suspended particles simultaneous to the electrical measurements; note the axes in the left corner for reference.

(Autolab PGSTAT 30), and redox reactions transfer the electric current to ionic current. ${ }^{24}$ The anode, where copper oxidizes, serves as a $\mathrm{Cu}^{2+}$ cation source, and the cathode, where copper reduces, acts as a $\mathrm{Cu}^{2+}$ cation sink, allowing for a steady-state current in the stagnant fluid. Two copper wires are inserted at both sides of the membrane $(0.6 \mathrm{~mm}$ from the membrane in the anode compartment and $7.3 \mathrm{~mm}$ from the membrane in the cathode compartment), acting as sense electrodes. Using a feedback loop, the voltage difference between the sense electrodes is kept at $\Delta V=1 \mathrm{~V}$ and the time-dependent current between the anode and cathode is measured, $\Delta I(t)$. Because the current is continuous, the time-dependent resistance between the sense electrodes can be determined as $R(t)=\Delta V / I(t)$.

Flow dynamics in the anode compartment are visualized by seeding the solution with $0.1 \mathrm{wt} \% 2 \mu \mathrm{m}$ red polystyrene tracer particles (Microparticles $\mathrm{GmbH}$, with a density of $1.05 \mathrm{~g} / \mathrm{cm}^{3}$ ). The particles 
are illuminated with a thin $(\approx 0.2 \mathrm{~mm})$ laser sheet $(808 \mathrm{~nm}$, Firefly, Oxford Lasers), see Figure 2, and the reflected light is captured through a long-distance magnifying lens $(2-7 \times$, Navitar) on a camera at $10 \mathrm{frames} / \mathrm{s}$. From the recorded particle displacements, the vector field is determined using particle image velocimetry ( $\mu \mathrm{PIV})$ analysis (DaVis, LaVision) following the same algorithms as described in our previous paper, ${ }^{25}$ see for example Figure 4 . The coordinate system is taken such that $x$ represents the direction perpendicular to the membrane, $y$ the direction along the membrane and in the plane of the illuminating laser sheet, and $z$ the depth into the cell, as denoted by the axes in Figure 2.

Concentration fields are measured using fluorescence lifetime image microscopy (FLIM), (LIFA, Lambert Instruments) by adding $2.5 \mu \mathrm{M}$ green-fluorescent dye (Alexa Fluor 488 Cadaverine, Life Technologies) to the solution. The fluorescence decay of the dye is indicative of the $\mathrm{CuSO}_{4}$ concentration in the range between 1 and $100 \mathrm{mM}$ (see the supporting information of ref 25 for the calibration curve and error estimation). A modulated blue light emitting diode (LED) light is sent through a $5 \times$ magnifying objective (Zeiss), and the fluorescence is captured on a $696 \times 520$ pixels CCD camera, with each pixel representing an area of $4.17 \times 4.17 \mu \mathrm{m}^{2}$. More details on the FLIM setup can be found in Chapter 2 of de Valença. ${ }^{23}$

Numerical Analysis. A theoretical framework based on PoissonNernst-Planck with the Navier-Stokes equations was used to investigate the coupling of the structure to potential, flow, and concentration fields in the system. Simulations of various structured membrane geometries were carried out by solving this framework numerically via the finite element method in COMSOL Multiphysics 5.2 , utilizing a $2 \mathrm{D}$ representation of the system for numerical simplification. Quadratic Lagrange elements were used to solve for the potential and the cation/anion concentration, whereas the Stokes equations were solved using P2-P1 (2nd order elements for velocity and 1st order for pressure). Mesh independence, at the assumed dimensionless Debye length, was achieved through local refinement near the membrane interface to resolve the regions of highest electricfield distortion. Simulations were run up to a dimensionless time of 2 (corresponding to $2500 \mathrm{~s}$ ), which was more than sufficient for achieving a stationary numerical solution in simulations.

The dimensionless formulation of the governing equations was taken as that given previously by ref 26 , the reader is referred to this and other work for further details ${ }^{18,27}$

Mass conservation-anion and cation

$$
\begin{aligned}
& \frac{\partial c^{ \pm}}{\partial t}=-\nabla \cdot \mathbf{j}^{ \pm} \\
& \mathbf{j}^{ \pm}=c^{ \pm} \mathbf{u}-\nabla c^{ \pm}-\mp c^{ \pm} \nabla \phi
\end{aligned}
$$

Navier-Stokes equations

$$
\begin{aligned}
& \frac{1}{S c} \frac{\partial \mathbf{u}}{\partial t}=-\nabla p+\nabla^{2} \mathbf{u}-\frac{\kappa}{2 \varepsilon^{2}}\left(c^{+}-c^{-}\right) \nabla \phi \\
& \nabla \cdot \mathbf{u}=0
\end{aligned}
$$

with the following terms

$$
\begin{aligned}
& \kappa=\frac{\varepsilon_{\mathrm{r}} \varepsilon_{0}}{\mu D}\left(\frac{k_{\mathrm{B}} T}{z e}\right)^{2} \\
& \varepsilon=\frac{\lambda_{\mathrm{D}}}{L}, \quad \lambda_{\mathrm{D}}=\sqrt{\frac{\varepsilon_{\mathrm{r}} \varepsilon_{0} k_{\mathrm{B}} T}{2(z e)^{2} c_{0}}} \\
& S c=\frac{\mu}{\rho D}
\end{aligned}
$$

Poisson equation

$$
-2 \varepsilon^{2} \nabla^{2} \phi=c^{+}-c^{-}
$$

Characteristic scales

$$
\begin{array}{cl}
t_{\text {diff }}=\frac{L^{2}}{D}, \quad v_{\text {diff }}=\frac{D}{L}, & p_{0}=\frac{\mu D}{L^{2}}, \\
V_{\mathrm{T}}=\frac{k_{\mathrm{B}} T}{z e}, & j_{0}=\frac{D c_{0}}{L}
\end{array}
$$

Membrane boundary conditions

$$
\mathbf{u}=0, \quad \phi=0, \quad c^{+}=c_{\text {mem. }}, \quad j_{\mathrm{y}}^{-}=0
$$

Reservoir wall boundary condition

$$
\mathbf{u}=0, \quad \phi=\Delta \phi, \quad c^{+}=c^{-}=1
$$

In eqs $1-8, c^{+}$and $c^{-}$represent cation and anion concentrations in the fluid, respectively, $j$ represents the ion flux, $\phi$ represents the potential, $\mathbf{u}$ represents the velocity vector field, and $p$ represents the pressure. The natural time-scaling in this formulation is against the Schmidt number $(S c)$, with other characteristic scales shown in eq 9. The boundary conditions at the membrane and the reservoir are given as eqs 10 and 11 .

For the potential distribution, the lower boundary was set at a constant potential (corresponding to the physically applied value of 1 $\mathrm{V}$ ), whereas the membrane interface was considered as ground. The cation concentration at the lower boundary was set at 1 , whereas it was fixed at 2 at the membrane interface, as per ref 26. For anions, both boundaries were set as no-flux, corresponding to the respective cationexchange interfaces. The fluid boundaries were no-slip at both upper and lower boundaries. The left and right boundaries correspond to noslip/no-flux conditions (walls of the system). The choice of the dimensionless scaling distance (relative size of the Debye length to the system size, $\epsilon$ ) was set at $10^{-3}$ for numerical stability. This choice was found previously to not strongly influence the solution, ${ }^{26,27}$ although it may be possible that this is no longer the case for the case with geometric structuring of the membrane. For the purposes of these simulations, which were to estimate orders of magnitude of velocity and concentration, as well as assess the coupling of the flow and electric behavior of the system, it was deemed to be an acceptable choice.

\section{RESULTS \& DISCUSSION}

Onset of EC. Each measurement starts by applying a constant voltage drop of $1 \mathrm{~V}$ between the copper electrodes on both sides of the membrane with an initial stagnant and homogeneous solution, $\mathbf{u}=0$ and $\nabla c(x, t)=0$. The initial Ohmic resistance $R_{\mathrm{ini}}=V /\left(i \cdot A_{\mathrm{mem}}\right)=5.0 \pm 0.2 \mathrm{k} \Omega$ does not depend on the membrane type. The initial resistance corresponds to the expected resistance based on the conductivity of $10 \mathrm{mM} \mathrm{CuSO}_{4}(\approx 1.5 \mathrm{mS} / \mathrm{cm})$ in the channel geometry, see Supporting Information. ${ }^{28}$

Because of the selectivity of the membrane and anode, cations migrate in and out the anode compartment, whereas the anions, migrating in the opposite direction, are blocked. The discontinuity in ion flux at the interfaces will lead to a change in ion concentration. This process is called ICP and can be directly observed from the change in fluorescence lifetime of the dye due to the change in $\mathrm{CuSO}_{4}$ concentration (see Figure 7 and Supporting Information Movie S1). Because the resistance is inversely proportional to the ion concentration, the resistance of the depleted side (anode side of the membrane) will dominate over the enriched side where the resistance decreases. The concentration difference at the two sides of the membrane also gives rise to a Nernst potential, as the membrane is ion selective. This potential is typically much smaller than the applied potential difference $\left(V_{\mathrm{n}}=\frac{k_{\mathrm{B}} T}{z e} \ln \left(c_{1} / c_{2}\right) \approx 67 \mathrm{mV}\right.$ for $c_{1}=20 \mathrm{mM}$ and $c_{2}=0.1$ $\mathrm{mM})$. 
a)

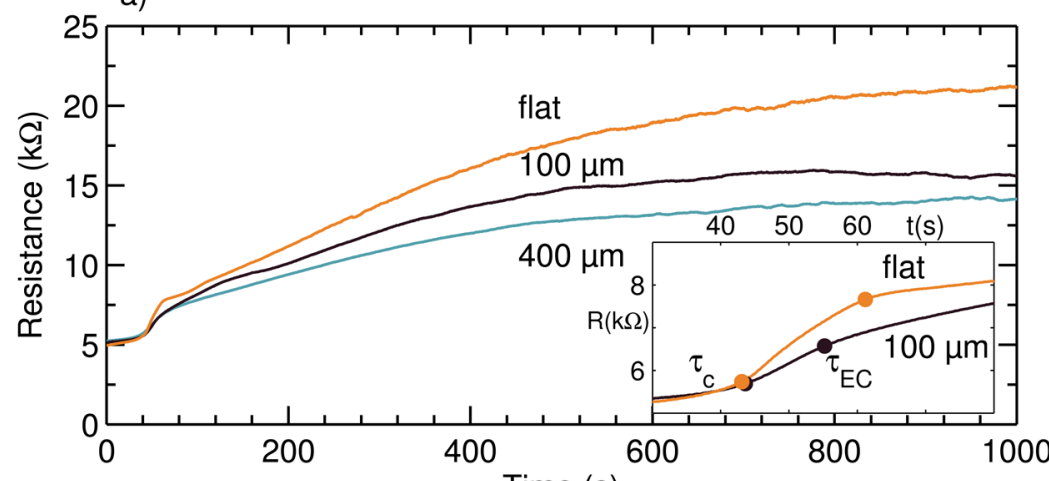

b)

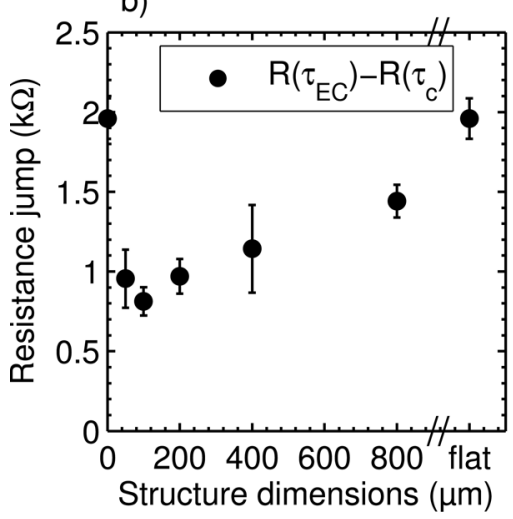

Time (s)

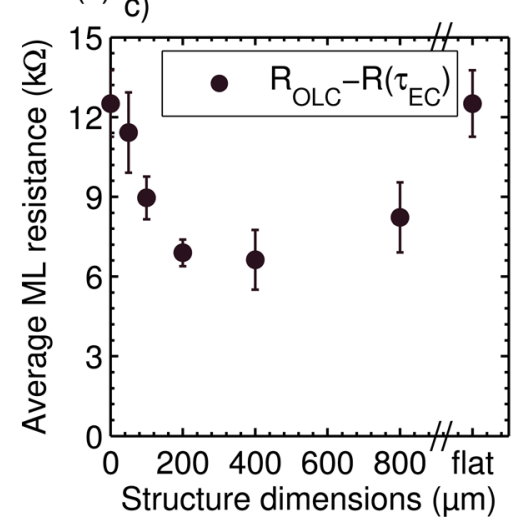

Figure 3. (a) Electrical resistance in time after applying $\Delta V=1 \mathrm{~V}$ for different membranes. The inset shows the onset of OLC, displaying the critical depletion time $\left(\tau_{\mathrm{c}}\right)$ and EC onset time $\left(\tau_{\mathrm{EC}}\right)$. (b) Resistance increase $\left(R\left(\tau_{\mathrm{EC}}\right)-R\left(\tau_{\mathrm{c}}\right)\right)$ indicates the threshold voltage drop needed to start EC. The value of the flat membrane has been placed at the beginning and the end of the $x$ axis, because it can be seen as either a zero or an infinite structure dimension. (c) Average ML resistance, calculated as the average resistance value over the last $400 \mathrm{~s}\left(R_{\mathrm{OLC}}\right)$ and subtracting $\left(R\left(\tau_{\mathrm{EC}}\right)\right)$, as a function of structure dimension. The error bars in $(b, c)$ represent the $95 \%$ confidence interval calculated from the mean values in the 3 to 6 repeated measurements with the same membrane.

When the concentration at the interface approaches zero, the resistance in the thin interfacial layer sharply increases, as seen in the inset in Figure 3a. ${ }^{6,27,29}$ This depletion layer is also seen in Figure $7 \mathrm{a}$. The depletion time, $\tau_{\mathcal{c}}$ is taken as the first point, where the rate of change of the resistance gradient is maximum $\left(\partial_{t}^{3} R(t)=0\right)$. The different membranes display very similar depletion times $\tau_{\mathrm{c}}=42 \pm 5 \mathrm{~s}$ and the corresponding resistance at depletion, $R\left(\tau_{\mathrm{c}}\right)=5.7 \pm 0.2 \mathrm{k} \Omega$. The second point is where $\partial_{t}^{3} R(t)=0$ marks the start of electroconvective mixing of the interface layer. This second transition time, $\tau_{\mathrm{EC}}$, coincides with the first particle motion at the membrane in the optical measurements (seen in Supporting Information Movie S4).

The increase in resistance, defined as $R\left(\tau_{\mathrm{EC}}\right)-R\left(\tau_{\mathrm{c}}\right)$, is less for the structured membranes compared to the unstructured membrane, see Figure $3 \mathrm{~b}$. The error bars represent the $95 \%$ confidence interval over three to six measurements in conditions with and without particles to visualize the flow. These results can be interpreted as a shortening of the plateau length in a typical $I V$ curve, as seen by Balster et al. ${ }^{19}$ and are in line with the numerical predictions of Rubinstein and Zaltzman. $^{10}$

Growth of EC. Structures on the membrane affect the onset of the electroconvective flow, as seen in Figure $4 a-c$. A detailed study on the development of the observed electroconvective vortices paves the way for an explanation to what type of mixing occurs. First, the general properties of the electroconvective mixing are described, followed by the difference between mixing at a flat membrane versus mixing at a structured membrane.

The layer with advection is called the ML, whereas the consequently shrinking stagnant layer is called the diffusion layer (DL). Such a two layer separation of the system has been observed before ${ }^{29}$ and also predicted numerically. ${ }^{26}$ The concentration profile obtained from FLIM experiments confirm that the DL contains a concentration gradient, whereas the ML has a lower constant concentration, $O(1 \mathrm{mM})$, see Figure 7 .

Once vortices become apparent $(\approx 0.3 \mathrm{~mm})$, the boundary of the $\mathrm{ML}$ is derived from the $\mu \mathrm{PIV}$ results, following the same algorithm as our previous work. ${ }^{29}$ First, the root mean square (rms) of the velocity vector values along the membrane (horizontal row) is determined. The vortex boundary is taken as the point where this rms velocity drops below $20 \%$ of the maximum rms velocity. This threshold value underestimates the ML thickness but is less prone to erroneous vectors. The position of the membrane is taken as the edge of the extrusion. Initially, the height of the ML and the average rms velocity within the layer grow linearly in time, see Figure 5. The resistance grows in a similar way, see Figure 3a, which indicates that the additional resistance primarily arises from a growing $\mathrm{ML}$ which has a low average concentration. It remains an open question if the voltage drop near the membrane surface remains constant while the vortices grow. In other words, It remains an open question if the initial resistance increase $\left(R_{\text {jump }}\right)$ is the same value although larger vortices are driven by electroosmotic coupling. 

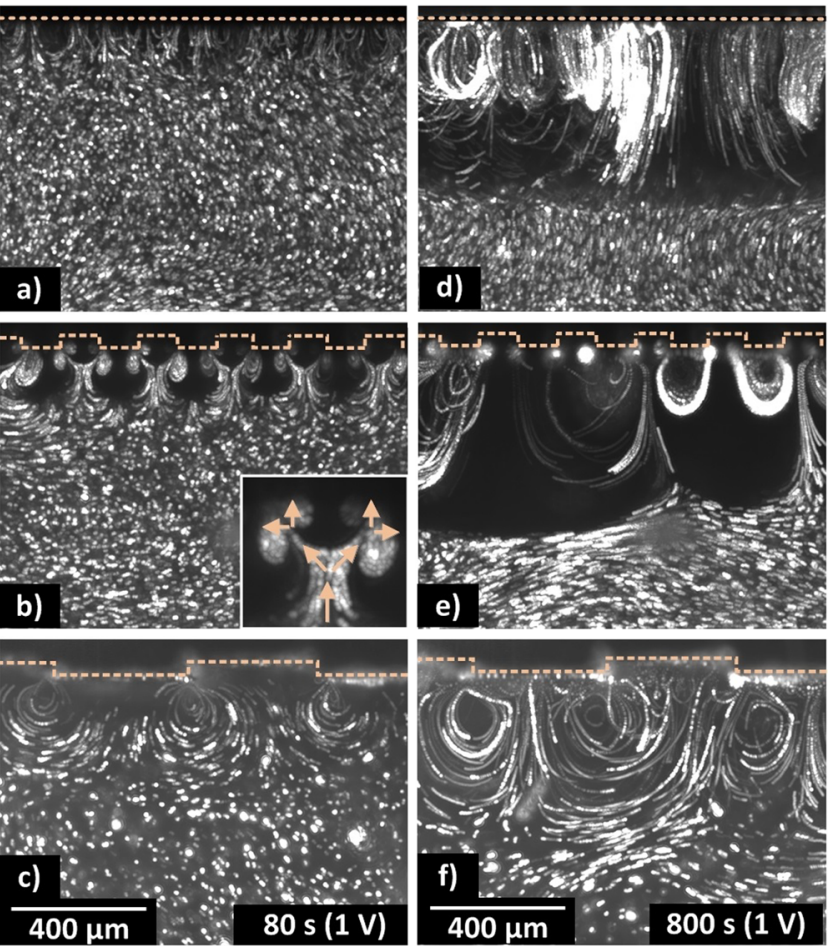

Figure 4. Overlaying the 100 images shows the particle pathlines over $10 \mathrm{~s}$ : (a) flat, (b) $100 \mu \mathrm{m}$, and (c) $400 \mu \mathrm{m}$ (70-80 s). During the growth phase, the structures influence the electroconvective vortex shape: (d) flat, (e) $100 \mu \mathrm{m}$, and (f) $400 \mu \mathrm{m}$ (790-800 s). At a flat membrane, the electroconvective vortices move along the membrane. At the membrane with ridges of $400 \mu \mathrm{m}$, the thickness of the vortex layer is bigger and the center point of the vortices is less mobile. At a membrane with $100 \mu \mathrm{m}$ ridges, voids empty of particles are formed, while the particle density at the surface increases locally. The membrane edge is indicated with a dotted line. The reader is referred to Figure 2 for the axes.

Near the flat membrane, the first particle motion (around $\left.\tau_{\mathrm{EC}}\right)$ occurs at random locations [see Supporting Information Movie S1, as well as the supporting movies in de Valença et al. (2015) and de Valença et al. $\left.(2017)^{25,29}\right]$. The particle pathlines reveal small vortices that grow and merge with other vortices while moving laterally along the surface. The rms velocity inside the ML grows linearly as well, similar to what was observed in the earlier experiments. ${ }^{29}$

Near the structured membranes, the vortices also grow and merge, but often, the vortices have preferred locations.
Repeating the experiments at different cross-section positions $(\Delta z \approx 1.5 \mathrm{~mm})$ shows similar behavior. This leads to the hypothesis that the $3 \mathrm{D}$ behavior of mixing occurs as (cylindrical) vortex rolls along the ridges. Typically, the flow is directed to the corners of the structures with the inflow from above the gap and the outflow above the extrusion. This is opposite to the theoretical prediction at sinusoidal curved membranes. ${ }^{10,15}$ At the structured membrane, typically two types of vortices appear, as shown in Figure $4 \mathrm{~b}$. The inset shows an inner vortex along the surface parallel to the electric field and an outer vortex along the surface perpendicular to the electric field. Most likely, the inner vortex is driven by the electro-osmotic wall flow, also referred to as the Dukhin mode. The outer vortices are probably driven by the EKI, also referred to as the Rubinstein mode. ${ }^{4}$

The inner vortices are visible as flow pockets where particles are trapped. The diameter of these vortices does not grow larger than $\approx 50 \mu \mathrm{m}$, and the velocities inside can reach 100 $\mu \mathrm{m} / \mathrm{s}$. The aggregation of particles, the presence of the membrane, and the optical resolution of the system make it challenging to determine the velocities with high precision. The opposite effect of trapping is the depletion of particles at the edge of the ML. Whether the particle motion deviates from the fluid streamlines and moves toward the vortex center depends on the body forces on the particle.

The outer vortices grow up to $\approx 0.5 \mathrm{~mm}$ and supply the depleted interface layer near the membrane with a higher concentrated solution. The width of the outer vortices is approximately the same as their height. When the width of the vortices is similar to the width of the structures, less mobile vortices are observed that are pinned to the membrane structure. If the vortex width grows larger than the structure dimension, the pinned vortex profile breaks down, Figure 4e. Vortices seem to hold their positions at the membrane between the ratio $L_{\text {mix }}=0.5 \cdot L_{\text {mem }}$, where they are squeezed ellipses along the membrane (Figure $4 \mathrm{c}$ ), and $L_{\text {mix }}=2 \cdot L_{\text {mem }}$, where they are elongated ellipses from the membrane (Figure $4 \mathrm{~b}$ ). When the vortices are spherical, $L_{\text {mix }} \approx L_{\text {mem }}$ the void area is the lowest. In the larger structures $(400$ and $800 \mu \mathrm{m})$ the vortices are located only at the corners of the structures. With the growth of the $\mathrm{ML}$, these vortices do not need to merge while growing to the saturated size.

There is a natural ratio between the vortex size and structure size, which keeps the vortices confined and stable. The lateral movement does not seem to enhance the ion transport toward the membrane. Confined vortices seem to be equally or more

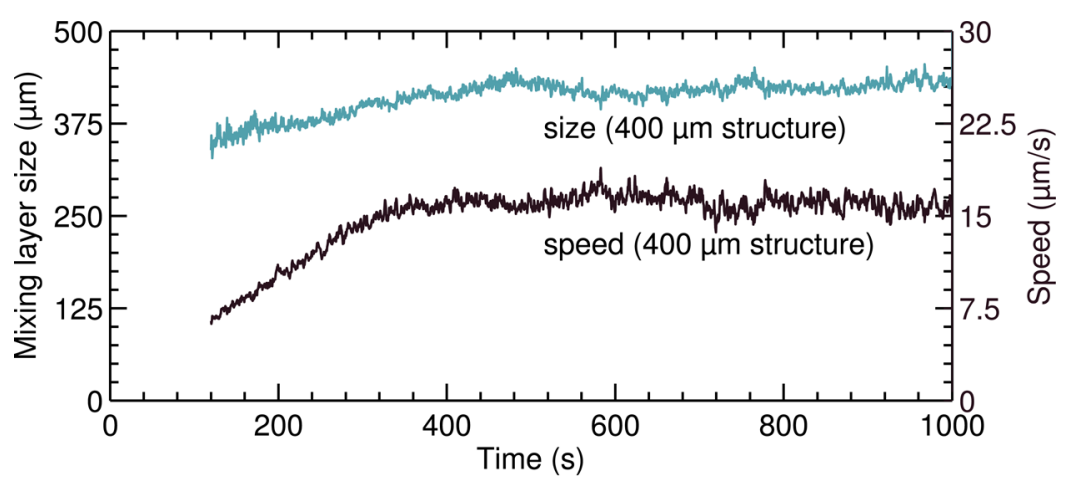

Figure 5. (a) ML thickness (vortex size) and average rms velocity within the ML (vortex speed) grow in time until a saturated size and speed is reached. 

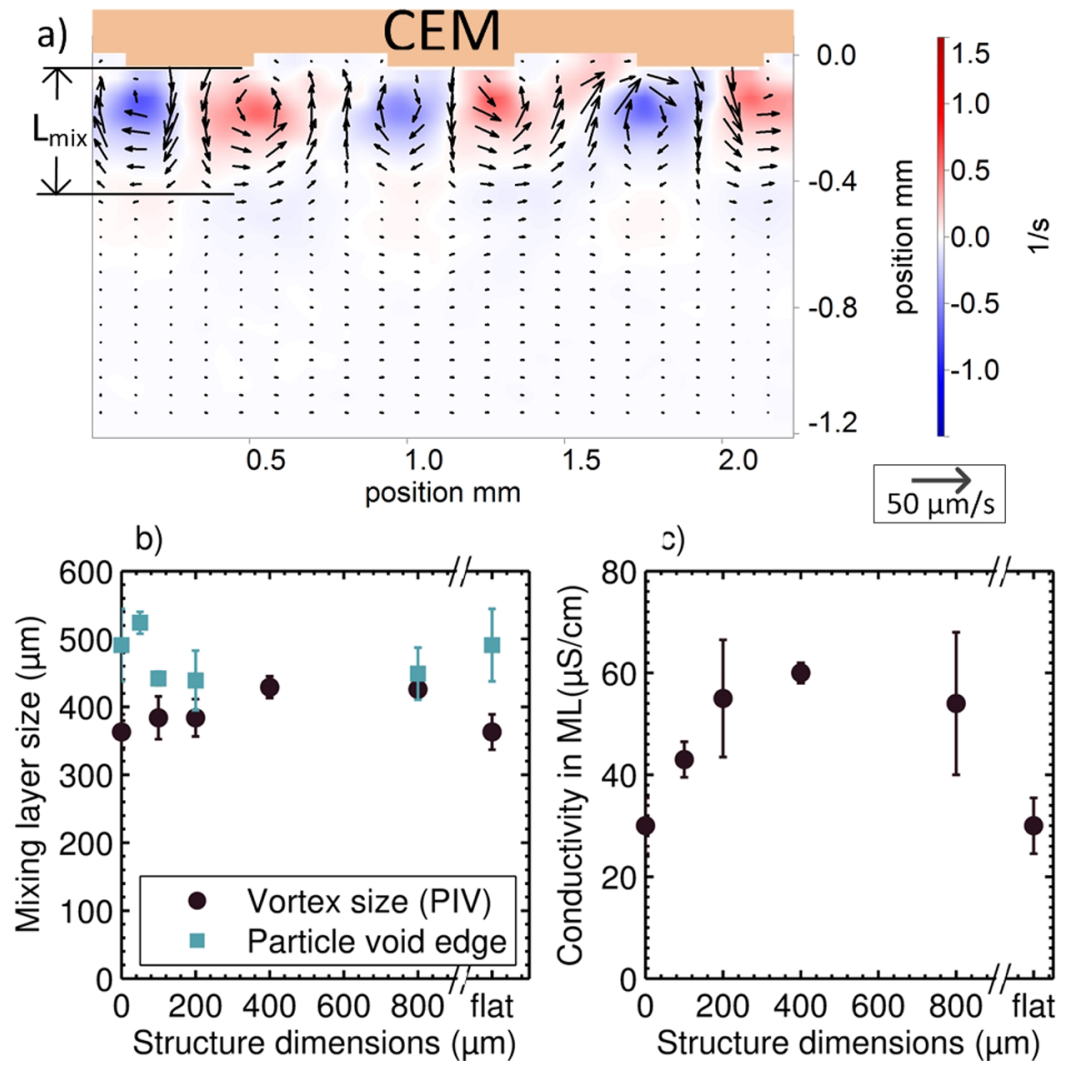

Figure 6. (a) Steady vortices at the $400 \mu \mathrm{m}$ are resolved with $\mu$ PIV. Shown is the average motion between 700 and $710 \mathrm{~s}$ (100 image pairs). The vortex boundary was taken as the place where the rms velocity was $20 \%$ of the maximum. $L_{\text {mix }}=426 \mu \mathrm{m}$ with $v_{\text {rms }}=17 \mu \mathrm{m} / \mathrm{s}$. The background color represents the vorticity of the vector field. Every second row of vectors is omitted for visibility. (b) ML size is determined from $\mu$ PIV (as describe above) and from the edge of the particle void (by taking the horizontal average of the light intensity). (c) Conductivity within the ML is calculated as $\sigma=L_{\text {mix }} /\left(R_{\text {sat }} \cdot A_{\text {mem }}\right)$. The ML size determined from the PIV measurements is used. The error bars in $(\mathrm{b}, \mathrm{c})$ represent the edges of the $95 \%$ confidence interval calculated from the mean values of 2 to 4 repeated measurements with the same membrane.

effective in mixing the high and low concentrations compared to the ones with the lateral motion, which matches the predictions found in the recent numerical work. ${ }^{18}$

Saturation of EC. The ML grows to $\approx 0.5 \mathrm{~mm}$ within the saturation time of $t \approx 600 \mathrm{~s}$, as can be seen from the particle pathlines, see Figure $4 \mathrm{~d}-\mathrm{f}$, and the $\mu \mathrm{PIV}$ results in Figure 6 . This layer is most stable when the structures are around 400 $\mu \mathrm{m}$ and the lateral movement is minimal.

The conductivity of the saturated ML is estimated via $\sigma=$ $L_{\text {mix }} /\left(R_{\text {sat }} \cdot A_{\text {mem }}\right)$, where $R_{\text {sat }}$ is the average resistance in the ML from $t=600 \mathrm{~s}$ to $t=1000 \mathrm{~s}$ and $L_{\text {mix }}$ is the average length in this period. The conductivity of the ML is higher for the structured membranes compared to flat membranes, whereas the ML thickness $(\sim 500 \mu \mathrm{m})$ is comparable for all membranes, see Figure $6 \mathrm{~b}, \mathrm{c}$. The observed average conductivity can be related to a concentration of $\approx 0.2 \mathrm{mM}$ of $\mathrm{CuSO}_{4}$, see ref 28 .

The formation of the particle-depleted regions complicates the extraction of the flow field; therefore, the vectors are interpolated or extrapolated from the regions with sufficient amount of particles. The edges of the membrane microstructures trap tracer particles, thereby decreasing the particle concentration in the outer vortices. This particle-free zone was used as a measure for estimating the ML thickness. The ML thickness was determined by horizontally averaging the pixel intensity and track at what distance from the membrane, the intensity sharply increases. A similar approach was used to determine the ML thickness from the vector field data. The ML size, as determined by both methods, is presented as a function of the structure dimension in Figure $6 \mathrm{~b}$. The ML for the $50 \mu \mathrm{m}$ structured membrane has a significant void formation, which hinders the extracting of reliable PIV vector fields. At the 400 $\mu \mathrm{m}$ membrane, there are almost no particle-free zones. In general, the microstructured membranes display slightly larger ML thicknesses compared to the flat membrane. Potentially, the buoyancy acting in this stabilizing direction is limiting the growth of the vortices, in accordance with recent experimental $^{25}$ and numerical ${ }^{30}$ work.

FLIM is used to obtain concentration profiles near the ion exchange membrane during the ion transport in the saturated regime (see Figure 7$)$. Time averaging $(t=600-1000 \mathrm{~s})$ of the concentration field shows that the presence of the structures hinders the lateral motion of the vortices. The flow toward the membrane is located near the gap, having a higher concentration, whereas the flow away from the membrane is located above the extrusion, where there is a lower concentration. A time-averaged vector field $(t=600-800 \mathrm{~s})$ from a separate $\mu \mathrm{PIV}$ measurement overlays the concentration profile in Figure $7 \mathrm{~b}$. This could not be accomplished for the flat membrane because the flow dynamics in that case had a higher degree of fluctuation. For the $100 \mu \mathrm{m}$ structured membranes, no lateral concentration gradients were observed; although, this possibly could be due to the limited temporal resolution of the FLIM system (approximately $15 \mathrm{~s}$ ).

Ion-Flux Calculations. The current, flow, and concentration data allow for estimating the electromigration and diffusive and advective fluxes in the system. A one-dimensional 

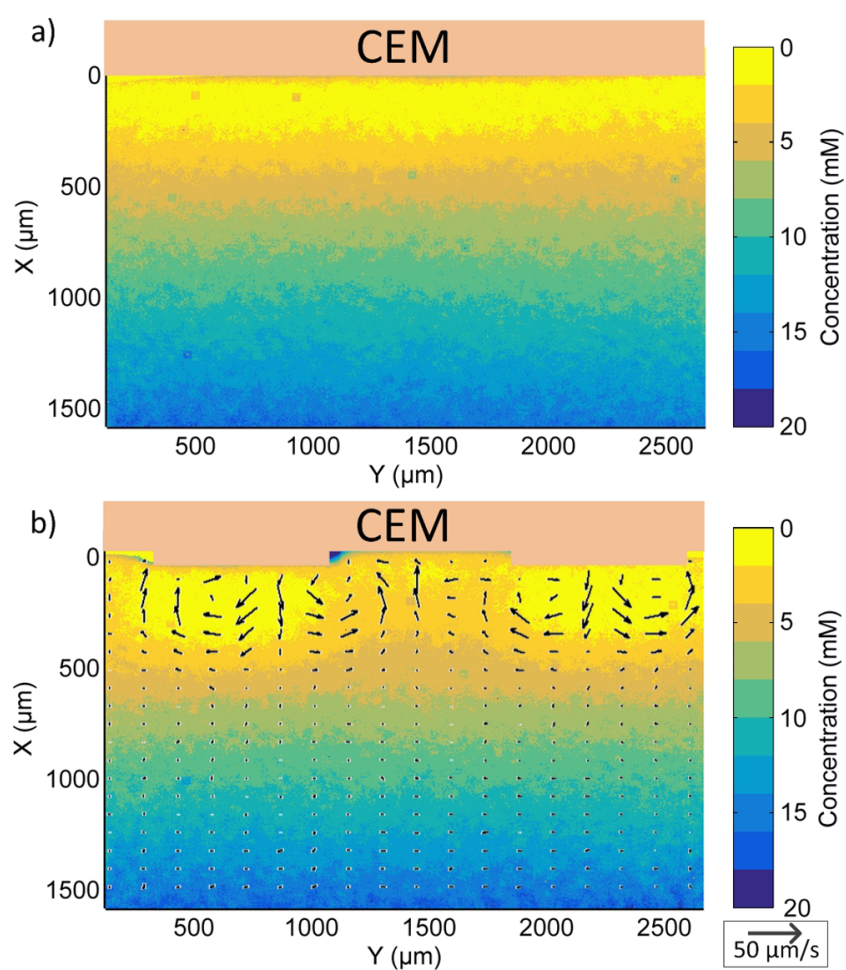

Figure 7. Concentration field (via FLIM) near a flat and structured $(800 \mu \mathrm{m})$ membrane in the saturated regime. The concentration field is a time-averaged field $(t=600-1000 \mathrm{~s})$, constructed from 33 separate lifetime images. On top of the concentration field at the 800 $\mu \mathrm{m}$ membrane, an overlay of the time-averaged velocity field (via $\mu \mathrm{PIV})$ is shown $(t=600-800 \mathrm{~s})$. The flow field is derived from a separate experiment with a similar electrical response. At the flat membrane also, an electroconvective $\mathrm{ML}$ is present, but because of the unsteady lateral motion of the vortices, the time-averaged flow field is not representative for the motion within the layer (see Supporting Information Movie S1).

(wall normal, $x$ direction) approach is taken to describe the indicated flux contributions. The current is assumed to be carried only by the $\mathrm{Cu}^{2+}$ and $\mathrm{SO}_{4}{ }^{2-}$, no $\mathrm{pH}$ gradient is assumed, and electroneutrality is assumed in the bulk. The ESCL is expected to be about $10 \times$ the Debye length ${ }^{14}$ in this system $\lambda_{\mathrm{D}}$ $\approx 10^{-8} \mathrm{~m}$. Numerical simulations showed that in the chaotic regime, charged regions do occur but that these regions do not significantly contribute to the overall ion advective transport. $^{26,27}$ The ion transport in $1 \mathrm{D}$ can be described as follows (see ref 8 for more details)

$$
\begin{aligned}
& J=J_{\mathrm{el}}+J_{\mathrm{diff}}+J_{\mathrm{adv}} \\
& J_{ \pm}=\frac{i t_{ \pm}}{z_{ \pm} F}-D \frac{\partial c_{ \pm}}{\partial x}+c_{ \pm} v
\end{aligned}
$$

where + represents $\mathrm{Cu}^{2+},-$ represents $\mathrm{SO}_{4}{ }^{2-}$, and the electric current density is given as $i=F\left(z_{+} J_{+}+z_{-} J_{-}\right)$. The transport number, $t_{ \pm}$, reflects the part of the current carried by each ion depending on its individual diffusion coefficient, $t_{ \pm}=D_{+} /\left(D_{+}+\right.$ $\left.D_{-}\right)$. Inside the liquid $t_{+}=0.4$ and $t_{-}=0.6$. An ideal CEM does not allow anions to pass, thus the $\bar{t}_{+}=1$ and $\bar{t}_{-}=0$. Faraday's constant is $F$, and $z_{+}=-z_{-}=2$ is the valence number of the ions. The ion concentration is $c_{+}$, and the total salt diffusion constant is $D=\left(z_{+}+\left|z_{-}\right|\right) D_{+} D_{-} /\left(z_{+} D_{+}+\left|z_{-}\right| D_{-}\right)=0.855 \times$ $10^{-9} \mathrm{~m}^{2} / \mathrm{s}^{24}$ The velocity of the liquid in the direction of the electric field is indicated by $v$.
This analysis focuses on the anion flux inside the anode compartment in the saturated regime. We discuss the flux both inside the stagnant layer (outside the vortex region) as well as inside the ML. Because the anions cannot pass the membrane and the anode, their total flux has to be zero. The FLIM concentration image (see Figure 7) shows a nearly linear concentration gradient along the electric field in the stagnant layer. The diffusive flux can be estimated to be $J_{\text {diff }}=-D \partial c / \partial x$ $=-D \cdot \Delta c_{\mathrm{DL}} / L_{\text {diff }}=1.1 \times 10^{-5} \mathrm{~mol} / \mathrm{m}^{2} \mathrm{~s}$, where $\Delta c_{\mathrm{DL}}=20 \mathrm{mM}$ and $L_{\text {diff }}=L_{\text {com }}-L_{\text {mix }}$. This corresponds to an electric current density of $3.5 \mathrm{~A} / \mathrm{m}^{2}$, whereas the measured current density lies between $5.3 \mathrm{~A} / \mathrm{m}^{2}$ (for the best observed case with structures) and $3.7 \mathrm{~A} / \mathrm{m}^{2}$ (for the flat membrane). With the growth of the $\mathrm{ML}$, the concentration gradient in the stagnant layer increases, enhancing the diffusive transport. For comparison, the theoretical limiting current density is $2.7 \mathrm{~A} / \mathrm{m}^{2}\left(\Delta c_{\mathrm{DL}}=20\right.$ $\mathrm{mM}$ and $L_{\text {diff }}=L_{\text {com }}=2 \mathrm{~mm}$ ).

For the transport in the ML, the diffusive contribution is neglected at first $\left(P e=v_{\text {rms }} L_{\text {mix }} / D \approx 7\right.$, with $v_{\text {rms }}=12 \mu \mathrm{m} / \mathrm{s}$, $L_{\text {mix }}=0.5 \mathrm{~mm}$ ). For simplicity, the $\mathrm{ML}$ is separated into a part with an upward flow, indicated as $J_{\mathrm{adv} \uparrow}=c_{\uparrow} v_{\uparrow}=6 \times 10^{-5} \mathrm{~mol} /$ $\mathrm{m}^{2} \mathrm{~s}$, and a part with downward flow, $J_{\mathrm{adv} \downarrow}=c_{\downarrow} v_{\downarrow}=-2 \times 10^{-5}$ $\mathrm{mol} / \mathrm{m}^{2} \mathrm{~s}$, where $v=20 \mu \mathrm{m} / \mathrm{s}$ (in either direction), $c_{\uparrow}=3 \mathrm{mM}$, and $c_{\downarrow}=1 \mathrm{mM}$. These values are derived from the $\mu \mathrm{PIV}$ and FLIM results, Figure $7 \mathrm{~b}$. This leads to $J_{\mathrm{adv}}=0.5 \times\left(J_{\mathrm{adv} \uparrow}+J_{\mathrm{adv} \downarrow}\right)$ $=2 \times 10^{-5} \mathrm{~mol} / \mathrm{m}^{2} \mathrm{~s}$. The orders of magnitude of the diffusive flux in the stagnant layer and the advective flux in the $\mathrm{ML}$ match and correspond with the imposed current density. This hints that at steady state, these are the dominant ion-transport processes.

Numerical Simulations. In the simulations, the iontransport development is calculated until a steady state occurs; the steady-state solution is shown in Figure 8. Before the steady state is obtained, two types of vortices are visible near the structure corner (see the Supporting Information for additional simulation images at earlier times). On top of the structure, two vortices appear, resulting in the flow directed toward the membrane in the middle of the extrusion, which are similar to the outer vortices in the experiment. Inside the gap, two vortices are also present, similar to the inner vortices observed in the experiments. However, the inner vortices grow out of the gap, over the structure corner, and become larger than the outer vortices. At steady state, the outer vortices have disappeared completely, resulting in the flow directed toward the membrane at the gap and away from the membrane above the extrusion. Despite the inverse direction compared to the experiments, the simulation results give the same orders of magnitude for velocity and concentration fields, as assessed by experiments. The results for the concentration are also in close agreement.

The precise mechanism of vortex formation and growth likely depends on many factors. For instance, the shape of the structure will influence the local electric-field profile both through changes in the local ion concentration profiles and from dielectric/conductivity contrast between the membrane and the medium. The structure shape was modeled with rounded edges with the same shape as observed in the scanning electron microscopy (SEM) images of the fabricated membranes. Still, the role of wall electro-osmosis is difficult to directly assess as the numerical resolution of the double layer representing the physical case $(10 \mathrm{~nm})$ is difficult for a flat membrane and the additional complication of structures only compounds the difficulty. The geometric structuring gives rise 

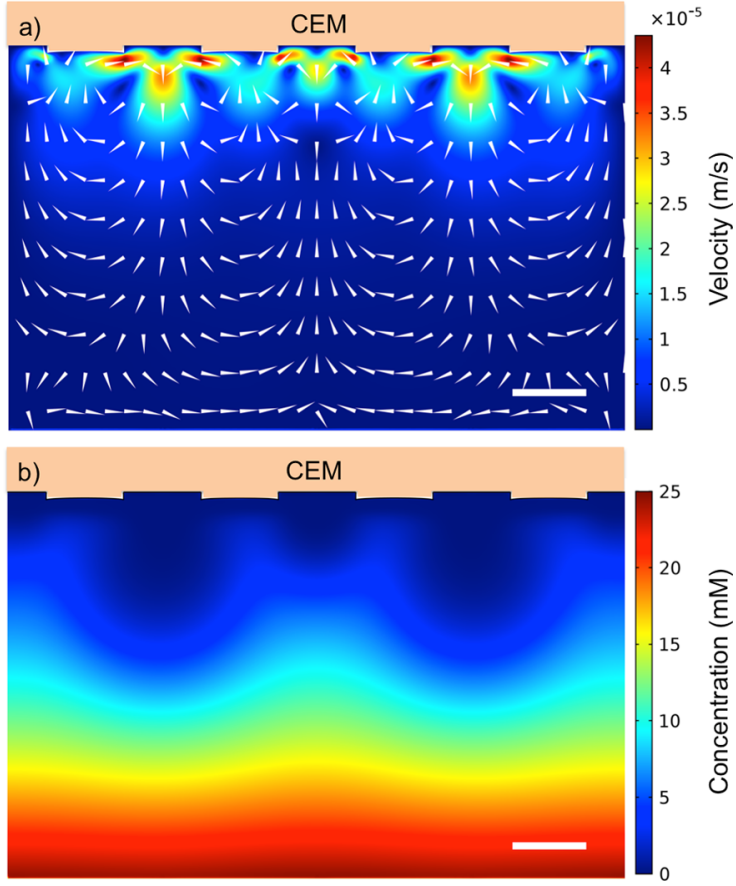

Figure 8. (a) Simulated flow profile at a membrane with $400 \mu \mathrm{m}$ structures in $10 \mathrm{mM} \mathrm{CuSO}_{4}$ with $\Delta V=0.5 \mathrm{~V}$ between the membrane interface and the opposite edge of the reservoir. (b) Simulated cation concentration profile for a membrane with $400 \mu \mathrm{m}$ structures after the simulations have achieved steady state. The scalebars represent 400 $\mu \mathrm{m}$.

immediately to a tangential electric field along the wall, purely because of the electrical property contrast between the medium and the membrane, and this can have a strong influence if not fully resolved. These type of roughness or curvature effects in the membrane are also difficult to assess as any significant geometric distortion could influence the direction and nature of the simulated EKI vortices and presumably also our experimental results. ${ }^{31}$

The electrohydrodynamic coupling constant in this case was approximately 0.14 for $\mathrm{CuSO}_{4}$, with a single diffusion coefficient of $0.855 \times 10^{-9} \mathrm{~m}^{2} / \mathrm{s}$ for both the anion and cation. It is also noted that because of the nature of the electrolyte balance in the system, there are potentially local $\mathrm{pH}$ gradients present that are not captured by the numerical model. This could influence the resulting vortex pattern as well as have affected the tracer particle zeta potentials during particletracking experiments. ${ }^{32,33}$ The vortices simulated by Davidson et al. $^{18}$ for patterned flat membranes are similar to the ones reported here. Their surface consists of a (flat) rectangular pattern with patches alternating between ion permeable and nonpermeable materials. The steady-state flow is directed toward the membrane above the nonconducting patch and away from the surface at the conducting patch. They also observed a range in which multiple steady-state situations are possible depending on initial perturbations, with larger vortices resulting in a lower resistance.

\section{CONCLUSIONS}

The dynamic behavior of electroconvective vortices at CEMs with line undulations during the OLC was quantified with electrical, flow, and concentration measurements. The presence of geometrical line structures on the membrane (undulations) decreases the energy needed to start and sustain EC. The start of EC is characterized by a sharp increase in the overall resistance, but it stabilizes with a significantly lower resistance for membranes with undulations compared to a flat membrane. The vortex growth is accompanied by a decrease in current (increase in resistance). The system reaches a dynamic equilibrium, described by a vortex $\mathrm{ML}(\mathrm{ML} \approx 0.5 \mathrm{~mm})$ and a stagnant $\mathrm{DL}(\mathrm{DL} \approx 1.5 \mathrm{~mm})$. From the electrical, the flow, and the concentration data, migration, advection, and diffusion of ions have been quantified, respectively. Advection and migration are dominant in the ML, whereas diffusion and migration are dominant in the DL. These results confirm the theoretical prediction that an undulated membrane surface enhances the OLC if the ML height is on the same order as the undulation width. This work demonstrates the potential for surface structure modification as it can greatly affect the ion transport, which is of fundamental interest and relevance for many applications.

\section{ASSOCIATED CONTENT}

\section{S Supporting Information}

The Supporting Information is available free of charge on the ACS Publications website at DOI: 10.1021/acs.langmuir.7b04135.

Conductivity calibration curve; reproducibility of measurements with the same membrane; simulation of the transient vortex; and details of supplemental movies (PDF)

Electroconvection at the flat membrane and $100 \mu \mathrm{m}$ and $400 \mu \mathrm{m}$ structured membranes; particle images of the vortex onset near the $100 \mu \mathrm{m}$ structured membrane; flow fields (every $5 \mathrm{~s}$ ) obtained with PIV analysis from the particle recordings at a $400 \mu \mathrm{m}$ structured membrane; and visualization of the concentration polarization using FLIM (ZIP)

\section{AUTHOR INFORMATION}

\section{Corresponding Author}

*E-mail: r.g.h.lammertink@utwente.nl.

ORCID

Jeffery A. Wood: 0000-0002-9438-1048

Notes

The authors declare no competing financial interest.

\section{ACKNOWLEDGMENTS}

This work was performed in the cooperation framework of Wetsus, European Centre of Excellence for Sustainable Water Technology (www.wetsus.eu). Wetsus is co-funded by the Dutch Ministry of Economic Affairs and Ministry of Infrastructure and Environment, the Province of Fryslân, the Northern Netherlands Provinces, and University Campus Fryslân. The authors would like to thank the participants of the research theme Biomimetic membranes for the discussions and financial support. This research was also financially supported by the European Research Council, under the ERC starting grant 307342-TRAM awarded to RGHL.

\section{REFERENCES}

(1) Rubinstein, S. M.; Manukyan, G.; Staicu, A.; Rubinstein, I.; Zaltzman, B.; Lammertink, R. G. H.; Mugele, F.; Wessling, M. Direct Observation of a Nonequilibrium Electro-Osmotic Instability. Phys. Rev. Lett. 2008, 101, 236101. 
(2) Fleury, V.; Chazalviel, J.-N.; Rosso, M. Theory and experimental evidence of electroconvection around electrochemical deposits. Phys. Rev. Lett. 1992, 68, 2492-2495.

(3) Kim, S. J.; Wang, Y.-C.; Lee, J. H.; Jang, H.; Han, J. Concentration Polarization and Nonlinear Electrokinetic Flow near a Nanofluidic Channel. Phys. Rev. Lett. 2007, 99, 044501.

(4) Nikonenko, V. V.; Kovalenko, A. V.; Urtenov, M. K.; Pismenskaya, N. D.; Han, J.; Sistat, P.; Pourcelly, G. Desalination at overlimiting currents: State-of-the-art and perspectives. Desalination 2014, 342, 85-106

(5) Rosso, M. Electrodeposition from a binary electrolyte: new developments and applications. Electrochim. Acta 2007, 53, 250-256.

(6) Strathmann, H. Ion Exchange Membrane Separation Processes, 1st ed.; Elsevier, 2004.

(7) Rosenberg, N. W.; Tirrell, C. E. Limiting Currents in Membrane Cells. Ind. Eng. Chem. 1957, 49, 780-784.

(8) Nikonenko, V. V.; Pismenskaya, N. D.; Belova, E. I.; Sistat, P.; Huguet, P.; Pourcelly, G.; Larchet, C. Intensive current transfer in membrane systems: Modelling, mechanisms and application in electrodialysis. Adv. Colloid Interface Sci. 2010, 160, 101-123.

(9) Moya, A.; Belashova, E.; Sistat, P. Numerical simulation of linear sweep and large amplitude ac voltammetries of ion-exchange membrane systems. J. Membr. Sci. 2015, 474, 215-223.

(10) Rubinstein, I.; Zaltzman, B. Electro-osmotically induced convection at a permselective membrane. Phys. Rev. E: Stat., Nonlinear, Soft Matter Phys. 2000, 62, 2238-2251.

(11) Bazant, M. Z.; Kilic, M. S.; Storey, B. D.; Ajdari, A. Towards an understanding of induced-charge electrokinetics at large applied voltages in concentrated solutions. Adv. Colloid Interface Sci. 2009, $152,48-88$.

(12) Dukhin, S. S. Electrokinetic phenomena of the second kind and their applications. Adv. Colloid Interface Sci. 1991, 35, 173-196.

(13) Mishchuk, N. A. Concentration polarization of interface and non-linear electrokinetic phenomena. Adv. Colloid Interface Sci. 2010, $160,16-39$.

(14) Rubinstein, I.; Zaltzman, B. Dynamics of extended space charge in concentration polarization. Phys. Rev. E: Stat., Nonlinear, Soft Matter Phys. 2010, 81, 061502.

(15) Demekhin, E. A.; Nikitin, N. V.; Shelistov, V. S. Direct numerical simulation of electrokinetic instability and transition to chaotic motion. Phys. Fluids 2013, 25, 122001.

(16) Karatay, E.; Druzgalski, C. L.; Mani, A. Simulation of chaotic electrokinetic transport: Performance of commercial software versus custom-built direct numerical simulation codes. J. Colloid Interface Sci. 2015, 446, 67-76.

(17) Chang, H.-C.; Demekhin, E. A.; Shelistov, V. S. Competition between Dukhin's and Rubinstein's electrokinetic modes. Phys. Rev. E: Stat., Nonlinear, Soft Matter Phys. 2012, 86, 046319.

(18) Davidson, S. M.; Wessling, M.; Mani, A. On the Dynamical Regimes of Pattern-Accelerated Electroconvection. Sci. Rep. 2016, 6, 22505 .

(19) Balster, J.; Yildirim, M. H.; Stamatialis, D. F.; Ibanez, R.; Lammertink, R. G. H.; Jordan, V.; Wessling, M. Morphology and microtopology of cation-exchange polymers and the origin of the overlimiting current. J. Phys. Chem. B 2007, 111, 2152-2165.

(20) Wessling, M.; Morcillo, L. G.; Abdu, S. Nanometer-thick lateral polyelectrolyte micropatterns induce macrosopic electro-osmotic chaotic fluid instabilities. Sci. Rep. 2014, 4, 4294.

(21) Pismenskaya, N. D.; Nikonenko, V. V.; Melnik, N. A.; Shevtsova, K. A.; Belova, E. I.; Pourcelly, G.; Cot, D.; Dammak, L.; Larchet, C. Evolution with time of hydrophobicity and microrelief of a cation-exchange membrane surface and its impact on overlimiting mass transfer. J. Phys. Chem. B 2012, 116, 2145-2161.

(22) Korzhova, E.; Pismenskaya, N.; Lopatin, D.; Baranov, O.; Dammak, L.; Nikonenko, V. Effect of surface hydrophobization on chronopotentiometric behavior of an AMX anion-exchange membrane at overlimiting currents. J. Membr. Sci. 2016, 500, 161-170.

(23) de Valença, J. C. Overlimiting current properties at ion exchange membranes. Ph.D. Thesis, University of Twente, 2017.
(24) Lide, D. E. CRC Handbook of Chemistry and Physics, 76th ed.; CRC Press, 1996; pp 5-90.

(25) de Valença, J. C.; Kurniawan, A.; Wagterveld, R. M.; Wood, J. A.; Lammertink, R. G. H. Influence of Rayleigh-Bénard convection on electrokinetic instability in overlimiting current conditions. Phys. Rev. Fluids 2017, 2, 033701.

(26) Druzgalski, C. L.; Andersen, M. B.; Mani, A. Direct numerical simulation of electroconvective instability and hydrodynamic chaos near an ion-selective surface. Phys. Fluids 2013, 25, 110804.

(27) Druzgalski, C.; Mani, A. Statistical analysis of electroconvection near an ion-selective membrane in the highly chaotic regime. Phys. Rev. E: Stat. Phys., Plasmas, Fluids, Relat. Interdiscip. Top. 2016, 1, 073601.

(28) Supporting Information.

(29) de Valença, J. C.; Wagterveld, R. M.; Lammertink, R. G. H.; Tsai, P. A. Dynamics of microvortices induced by ion concentration polarization. Phys. Rev. E: Stat., Nonlinear, Soft Matter Phys. 2015, 92, 031003.

(30) Karatay, E.; Andersen, M. B.; Wessling, M.; Mani, A. Coupling between Buoyancy Forces and Electroconvective Instability near IonSelective Surfaces. Phys. Rev. Lett. 2016, 116, 194501.

(31) Benneker, A. M.; Wood, J. A.; Tsai, P. A.; Lammertink, R. G. H. Observation and experimental investigation of confinement effects on ion transport and electrokinetic flows at the microscale. Sci. Rep. 2016, 6, 37236 .

(32) Femmer, R.; Mani, A.; Wessling, M. Ion transport through electrolyte/polyelectrolyte multi-layers. Sci. Rep. 2015, 5, 11583.

(33) Russel, W. B.; Saville, D. A.; Schowalter, W. R. Colloidal Dispersions; Cambridge University Press, 1989. 